Übersichten

Nervenarzt 2020 $\cdot 91: 832-842$

https://doi.org/10.1007/s00115-020-00907-y

Online publiziert: 16. April 2020

(c) Der/die Autor(en) 2020

\author{
Ayda Rostamzadeh ${ }^{1}$ Frank Jessen ${ }^{1,2}$ \\ ' Klinik für Psychiatrie und Psychotherapie, Uniklinik Köln, Medizinische Fakultät, Köln, Deutschland \\ ${ }^{2}$ Deutsches Zentrum für Neurodegenerative Erkrankungen (DZNE), Bonn, Deutschland
}

\title{
Früherkennung der Alzheimer- Krankheit und Demenzprädiktion bei Patienten mit leichter kognitiver Störung
}

\section{Zusammenfassung aktueller Empfehlungen}

\section{Hintergrund}

\section{Durch die Fortschritte in der Früher- kennung der Alzheimer-Krankheit („Alzheimer's disease“, AD) sowie der Prädiktion der Alzheimer-Demenz werden hierfür eingesetzte diag- nostische und prädiktive Verfahren zunehmend klinisch bedeutsam. Auch führt der wissenschaftliche Fortschritt zu einem verstärkten Bewusstsein um die Früherken- nung der $A D$ in der Bevölkerung und entsprechende diagnostische Angebote werden immer häufiger aktiv nachgefragt. Dies steht vor dem Hintergrund, dass die AD bisher nicht heilbar ist und symptomati- sche medikamentöse Ansätze im Stadium der Alzheimer-Demenz ei- ne nur begrenzte Verzögerung der Krankheitsprogression bewirken können.}

Insbesondere bei der leichten kognitiven Störung („mild cognitive impairment", MCI) werden Patienten, Angehörige und Ärzte mit der Frage der Frühdiagnostik der AD und der Demenzprädiktion konfrontiert. Unter MCI wird eine objektivierbare kognitive Beeinträchtigung bei im Wesentlichen intakten Alltagskompetenzen verstanden [1]. Das MCI-Stadium geht mit einem erhöhten Risiko für die Entwicklung einer Demenz einher. In jüngster Zeit sind durch Fortschritte im Bereich der Biomarkerent- wicklung und deren Einsatz in aussagekräftigen longitudinalen Kohortenstudien für MCI-Patienten individuelle Vorhersagen zum Demenzrisiko möglich geworden. Durch die Anwendung von Biomarkern kann zum einen die Alzheimer-Pathologie bei MCI-Patienten nachgewiesen werden. Zum anderen ermöglicht sie, eine Differenzierung der MCIRisikogruppe in jene mit einem geringen, mit einem mittleren und mit einem hohem Risiko für den Übergang in eine Demenz vorzunehmen [19, 52]. Bei einer ausschließlich biomarkerbasierten Risikovorhersage ist die Spezifität für die individuelle Prädiktion jedoch etwas eingeschränkt, da wichtige klinische Daten wie Alter und Geschlecht nicht in die Prognoseberechnung miteinfließen. Beispielsweise ist der Zusammenhang zwischen Alter, kognitiver Verschlechterung und der Amyloid-Pathologie nicht linear, sondern erreicht im hohen Lebensalter eine Sättigung [26]. Insofern sind für eine individuelle Risikoprädiktion neben der durch Biomarker nachgewiesenen Alzheimer-Pathologie weitere klinische Variablen zu berücksichtigen, für die es jedoch zum derzeitigen Stand keine ausreichenden Validierungen im Rahmen von Studien gibt.

Die Prädiktion einer noch nicht heilbaren Erkrankung wie der Demenz bei Alzheimer-Krankheit wirft für Patienten und Angehörige viele Fragen auf. Sie bringt für Ärzte neue Herausforderungen in der Beratung über die biomar- kerbasierte AD-Früherkennung und der Abschätzung des Demenzrisikos anhand individueller Ergebnisse mit sich. Der folgende Artikel trägt internationale Empfehlungen für den Einsatz von Liquorbiomarkern zur Früherkennung der AD und der Demenzprädiktion bei Personen mit MCI zusammen. Die wesentliche Grundlage hierfür sind die Kriterien des National Institute on Aging und der Alzheimer's Association (NIA-AA), einer Expertenkonsensuspublikation, welche im Rahmen des europäischen BIOMARKAPD-Projektes (Joint Neurodegenerative Disease Research, JPND) publiziert wurde [21] sowie neue Empfehlungen einer multidisziplinären Arbeitsgruppe der Alzheimer's Association zum Einsatz von Liquorbiomarkern für die Diagnostik der Alzheimer-Krankheit [48]. Weitere thematisch verwandte Publikationen wurden zusätzlich herangezogen.

\section{"Mild cognitive impairment"}

Bei MCI handelt es sich um ein Syndrom zwischen "gesundem Altern" und der frühen Demenz, wobei im Verlauf von MCI eine Verbesserung der kognitiven Fähigkeiten mit vollständiger Remission ebenso wie ein Fortschreiten zur Demenz möglich sind [43]. Das MCISyndrom ist daher ein Risikostadium für eine Demenz und kann das Prodromalstadium einer Demenz bei AD oder anderer neurodegenerativer Erkrankungen darstellen. Man kann das 


\section{Tab. 1 NIA-AA-Kriterien für MCI bei Alzheimer-Krankheit (2011; [1])}

\section{Klinische Kriterien für MCl bei Alzheimer-Krankheit}

Kognitive Verschlechterung beobachtet durch den Betroffenen, durch einen Informanten oder durch einen Arzt

Einschränkungen in einer oder mehrerer kognitiver Domänen, typischerweise das Gedächtnis betreffend

Aktivitäten des täglichen Lebens erhalten

Keine Demenz

Zusätzliche Kriterien, die die Diagnose MCI bei Alzheimer-Krankheit stützen

Ausschluss einer symptomatischen Ursache (vaskulär, traumatisch oder andere medizinische) der kognitiven Verschlechterung, wenn möglich

Nachweis einer längsschnittlichen kognitiven Verschlechterung, wenn möglich

Genetische Vorbelastung im Sinne familiärer Alzheimer-Krankheit, wenn relevant

\section{Tab. 2 Einteilung der Biomarker für die Alzheimer-Krankheit. (Mod. nach [23, 24])}

\begin{tabular}{ll}
$\begin{array}{ll}\text { A Marker für die } \\
\text { Amyloid-Pathologie }\end{array}$ & $\begin{array}{l}\beta \text {-Amyloid } 1 \text {-42 } \text { bzw. die } \beta \text {-Amyloid }_{42 / 40} \text {-Ratio im Liquor } \\
\text { Amyloid-Deposition im Amyloid-PET }\end{array}$ \\
$\begin{array}{ll}\text { T Marker für die } \\
\text { Tau-Pathologie }\end{array}$ & $\begin{array}{l}\text { PTau-Protein im Liquor } \\
\text { Tau-Deposition im PET (derzeit nur für Forschungszwecke) }\end{array}$ \\
$\begin{array}{ll}\text { Neurodegeneration } \\
\text { tTau-Protein in Liquor }\end{array}$ \\
& $\begin{array}{l}\text { Mediale Temporallappen-/Hippokampusatrophie bzw. Reduktion der } \\
\text { kortikalen Dicke (AD-Signatur) im MRT }\end{array}$ \\
& $\begin{array}{l}\text { Hypometabolismus im medialen Parietallappen (Präkuneus), in den } \\
\text { temporoparietalen bzW. den frontalen Hirnarealen im }{ }^{18} \text { [F] FDG-PET }\end{array}$ \\
\hline $\begin{array}{l}\text { pTau phosphoryliertes Tau, tTau gesamtes Tau, MRT Magnetresonanztomographie, PET Positro- } \\
\text { nenemissionstomographie, FDG Fluordesoxyglucose }\end{array}$
\end{tabular}

MCI-Syndrom anhand der domänenspezifischen kognitiven Einschränkungen differenzieren in ein amnestisches MCI mit im Vordergrund stehender Gedächtnisstörung und ein nichtamnestisches MCI, bei dem nicht das Gedächtnis betreffende kognitive Einschränkungen dominieren (z.B. Sprache, Exekutivfunktion, Visuokonstruktion etc.; [43]). Die metaanalytische MCI-Prävalenz bei Personen $\geq 60$ Jahren liegt in der Allgemeinbevölkerung bei ca. $14-16 \%$ und in Kollektiven aus Gedächtnisambulanzen bei ca. $16-19 \%$ [22, 44, 45]. Das MCI-Syndrom geht mit einem erhöhten Risiko für eine Alzheimer-Demenz von etwa 33\% in 3 Jahren einher [38]. In einer Metaanalyse konnten mittlere jährliche Übergangsraten vom MCIStadium in eine Alzheimer-Demenz von $6,8 \%(95 \%-\mathrm{CI}=1,9-14,5 \%)$ in der Allgemeinbevölkerung und von $8,1 \%$ $(95 \%-\mathrm{CI}=6,3-10,0 \%)$ in spezialisierten Gedächtnisambulanzen nachgewiesen werden [38]. Limitierend ist, dass die der Metaanalyse zugrunde liegenden Studien aufgrund heterogener Untersu-

\section{Biomarker für die Alzheimer- Krankheit}

Die AD wird definiert durch pathophysiologische Veränderungen, die sich in vivo durch Biomarker und autoptisch durch histologische Untersuchungen nachweisen lassen [4]. Neuropathologische Korrelate der AD sind extrazelluläre Ablagerungen des Amyloid- $\beta_{1-42}$-Proteins und intrazelluläre Ablagerungen des phosphorylierten Tau-Proteins (pTau; [5]).

Die Unterteilung der Biomarker erfolgte zunächst in die Gruppe der Marker für die Amyloid-Pathologie (A) und die Marker für die Neurodegeneration (N; [25]). Basierend auf dieser Einteilung wurden in der NIA-AA-Konsensuspublikation aus dem Jahr 2011 die Wahrscheinlichkeiten angegeben, mit denen ein $\mathrm{MCI}$ durch eine $\mathrm{AD}$ verursacht wird („likelihood of MCI due to AD“; [1]). Bei einem Nachweis pathologischer Amyloid- und Tau-Biomarker besteht eine hohe Wahrscheinlichkeit für eine zugrunde liegende AD. Sind hingegen entweder nur die Amyloid-Biomarker oder nur die Tau-Biomarker pathologisch, besteht eine mittlere Wahrscheinlichkeit und bei unauffälligen Amyloid- als auch TauBiomarkern besteht eine geringe Wahrscheinlichkeit für das Vorhandensein einer AD [1].

Die durch Jack und Kollegen im Jahr 2016 eingeführte Neukonzeption der AD-Biomarker unterscheidet zwischen Biomarkern für die Amyloid-Pathologie (A), für die Tau-Pathologie (T) und für die Neurodegeneration (N; • Tab. 2; $[23,25])$. Bei der binären $\mathrm{A} / \mathrm{T} / \mathrm{N}-\mathrm{Klassi}$ fikation wird jeder Biomarker mit pathologisch (+) oder nichtpathologisch (-) klassifiziert [24]. Basierend hierauf wurde im Jahr 2018 durch die NIA-AAArbeitsgruppe eine Neukonzeption der $\mathrm{AD}$ für die Forschung vorgestellt, die die Alzheimer-Krankheit vollständig über Biomarker definiert und die Symptomatik als Stadium der Erkrankung wertet [24]. Diese Neukonzeption befindet sich aktuell in der wissenschaftlichen Evaluation und wird für die klinische Anwendung bisher nicht empfohlen. 
Nervenarzt 2020 $91: 832-842$ https://doi.org/10.1007/s00115-020-00907-y

(c) Der/die Autor(en) 2020

\section{A. Rostamzadeh · F. Jessen}

\section{Früherkennung der Alzheimer-Krankheit und Demenzprädiktion bei Patienten mit leichter kognitiver Störung. Zusammenfassung aktueller Empfehlungen}

\section{Zusammenfassung}

Die leichte kognitive Störung (, mild cognitive impairment", $\mathrm{MCl}$ ) zeichnet sich durch kognitive Einschränkungen bei im Wesentlichen erhaltener Alltagskompetenz aus. $\mathrm{MCl}$ ist ein Risikozustand für die Entwicklung einer Demenz und kann ein Prodromalstadium der Demenz bei Alzheimer-Krankheit („Alzheimer's disease“, AD) sein. Die AD ist durch zerebrale Ablagerungen von Amyloidund Tau-Aggregaten definiert und kann durch Biomarker für diese Veränderungen in vivo detektiert werden. Durch die Fortschritte in der biomarkerbasierten Früherkennung der $A D$, ist es möglich zwischen $\mathrm{MCl}-$ Patienten mit und ohne AD-Pathologie und somit zwischen Patienten mit einem geringen und einem hohen Risiko für die
Entwicklung einer späteren Demenz zu unterscheiden. Für die biomarkerbasierte Früherkennung der AD im MCl-Stadium gibt es bisher in Deutschland keine differenzierten Leitlinienempfehlungen. In dem vorliegenden Artikel werden die Empfehlungen einer europäischen Expertenkonsensuspublikation sowie einer multidisziplinären Arbeitsgruppe der Alzheimer's Association zum Einsatz von Liquorbiomarkern für die Diagnostik der Alzheimer-Krankheit bei MCI-Patienten zusammengefasst. Sind die klinischen Diagnosekriterien eines $\mathrm{MCl}$ anhand der klinischen Untersuchung und neuropsychologischen Testung erfüllt, wird empfohlen weiterführende Diagnostik (Blutuntersuchung, zerebrale Bildgebung) durchzuführen, um die differen- zialdiagnostische Einordnung zu präzisieren. Vor der Liquorbiomarkeruntersuchung sollte eine umfassende Beratung zu den Möglichkeiten, Grenzen und Risiken der biomarkerbasierten Früherkennung der AD und der Demenzprädiktion durchgeführt werden. Die Informationen über das individuelle Risiko für eine Demenz können sich bei Patienten und ihren nahestehenden Personen auf das psychische Wohlbefinden und die weitere Lebensplanung auswirken, daher werden klinische Verlaufsuntersuchungen empfohlen.

Schlüsselwörter

Mild cognitive impairment - AlzheimerKrankheit · Liquorbiomarker - Risikostadium . Prädiktion

\section{Early detection of Alzheimer's disease and dementia prediction in patients with mild cognitive impairment. Summary of current recommendations}

\section{Abstract}

Mild cognitive impairment (MCl) is characterized by cognitive deficits but essentially preserved competence in activities of daily living. It is a risk factor for the development of dementia and can reflect a prodromal predementia state of Alzheimer's disease (AD). The pathology of $A D$ is defined by cerebral deposition of amyloid-beta-1-42 protein and aggregation of phosphorylated tau protein, which can be identified in vivo by biomarkers for these alterations. As a result of advances in the field of biomarker-based early detection of $A D$, it is possible to differentiate between $\mathrm{MCl}$ patients with and without a pathological $A D$ condition and therefore, between patients with a low and those with a high risk for the development of dementia. At present there are no specific guideline recommendations in Germany for the diagnostic use of biomarkers in predementia detection of $A D$ and for dementia risk assessment in patients with $\mathrm{MCl}$. This article summarizes the current recommendations of a European expert consensus publication and a multidisciplinary working group of the Alzheimer's Association on the clinical application of cerebrospinal fluid (CSF) biomarkers for the diagnostics of $A D$ in patients with $\mathrm{MCl}$. If the clinical diagnostic criteria for $\mathrm{MCl}$ are fulfilled according to the medical history and neuropsychological testing, it is recommended to carry out further diagnostics (blood test, brain imaging) in order to more precisely define the differential diagnostic classification. Counseling on the potential benefits, limits and risks of biomarker testing for early $A D$ detection and dementia risk prediction should always precede assessment of CSF biomarkers. Information about the individual risk of developing dementia has potential consequences for the psychological wellbeing and life planning; therefore, clinical follow-up visits are recommended.

\section{Keywords}

Mild cognitive impairment · Alzheimer's disease - CSF-biomarkers - At-risk state . Prediction

\section{Marker für die Amyloid-Pathologie}

Für die Erfassung der Amyloid-Pathologie können im Liquor das $\beta$-Amyloid ${ }_{1-42}$

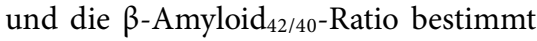
werden. Die $\beta$-Amyloid ${ }_{42 / 40}$-Ratio kann im Vergleich zum $\beta$-Amyloid A-42 $_{1 \text { de }}$ Sensitivität und Spezifität der Detektion einer $\mathrm{AD}$ verbessern [32]. Hierbei zeigt ein Quotient von $<0,8-1,0$ eine AmyloidPathologie an [31]. Eine weitere Möglichkeit ist die Darstellung der zerebralen
Amyloiddeposition mittels Amyloid-Positronenemissionstomographie(PET)Tracern, von denen mittlerweile mehrere zur Verfügung stehen. Zahlreiche Studien haben eine Korrelation zwischen der zerebralen Amyloid-Tracer-Bindung und einer erniedrigten $\beta$-Amyloid ${ }_{1-42^{-}}$ Konzentration bzw. $\beta$-Amyloid ${ }_{42 / 40}-\mathrm{Ra}-$ tio im Liquor nachweisen können [11, 31].

\section{Marker für die Tau-Pathologie}

Die Tau-Pathologie der $\mathrm{AD}$ lässt sich durch die Messung des phosphorylierten Tau-Proteins (pTau) im Liquor darstellen. Für die Erfassung der zerebralen Tau-Deposition gibt es Tau-PET-Tracer, die derzeit noch in der Entwicklungsphase sind. Erste Studien zeigen eine positive Korrelation zwischen zerebraler Tau-Deposition gemessen mit TauPET und pTau- sowie tTau-Konzentra- 
Tab. 3 Demenzprädiktion im MCl-Stadium anhand von Liquorbiomarkerprofilen. (Mod. nach [52])

\begin{tabular}{|c|c|c|c|}
\hline Amyloid-Marker & $\begin{array}{l}\text { pTau- und/oder tTau-Mar- } \\
\text { ker }\end{array}$ & $\begin{array}{l}\text { Risiko für eine Alzheimer-Demenz } \\
\text { innerhalb von } 3 \text { Jahren in \% }\end{array}$ & $\begin{array}{l}\text { Risiko für eine Alzheimer-Demenz } \\
\text { innerhalb von } 5 \text { Jahren }^{\mathrm{a}} \text { in } \%\end{array}$ \\
\hline Nicht pathologisch & Nicht pathologisch & Ca. 5 & Ca. 10 \\
\hline Pathologisch & Pathologisch & Ca. 60 & Ca. 90 \\
\hline Pathologisch & Nicht pathologisch & Ca. 20 & Ca. 45 \\
\hline Nicht pathologisch & Pathologisch & Ca. 25 & Ca. 50 \\
\hline
\end{tabular}

tionen im Liquor sowie dem histopathologischen Tau-Nachweis [8, 14, 49]. Da die Dateninterpretation der Tau-Tracer, auch in Bezug auf Spezifität der Anreicherung, wesentlich komplexer als die der Amyloid-Tracer ist, sind noch weitere Entwicklungen notwendig, ehe sie klinische Anwendung finden werden.

\section{Marker für die Neurodegeneration}

Die Biomarker für die Neurodegeneration stellen Indikatoren eines Nervenzellverlustes dar und sind nicht spezifisch für eine $\mathrm{AD}$. Das Gesamt-Tau-Protein (tTau) im Liquor weist auf einen Nervenzellverlust und eine Neurodegeneration hin und korreliert bei der AD mit der pTau-Konzentration im Liquor [32]. Mittels struktureller Magnetresonanztomographie (MRT) kann das Atrophiemuster beurteilt werden. Insbesondere die mediale Temporallappenatrophie gilt als typisch für die Neurodegeneration bei $\mathrm{AD}$, jedoch ist sie nicht spezifisch hierfür [12]. Neuere Studien beschreiben ein AD-typisches Muster der Reduktion der kortikalen Dicke, welches ebenfalls als ein MRT-Marker für die Neurodegeneration bei $\mathrm{AD}$ verwendet werden kann [24]. Mittels FDG-PET kann der zerebrale Glukosemetabolismus dargestellt werden, der auf die synaptische Dysfunktion und das Ausmaß der Neurodegeneration hinweist. Für die AD typisch sind ein Hypometabolismus im medialen Parietallappen (Präkuneus) und in den temporoparietalen sowie frontalen Hirnarealen [39].

\section{Chronologisches Modell der Alzheimer-Pathologie}

Ein hypothetisches Modell für die Chronologie der pathophysiologischen Ver- änderungen im Verlauf der AD wurde von Jack und Kollegen im Jahr 2013 vorgeschlagen [25]. Hierbei beginnt die Erkrankung Jahrzehnte vor Symptombeginn mit einer Amyloiddeposition. Dem Modell folgend können als erstes die Amyloidablagerungen durch Liquor- oder PET-Untersuchungen bereits im präklinischen Stadium nachgewiesen werden. Die Ausbreitung der Tau-Pathologie erfolgt dann über die medialen Temporallappen hinaus [5] und sowohl die tTau- als auch die pTau-Konzentrationen im Liquor werden im Verlauf dieser Ausbreitung auf andere Gehirnregionen pathologisch. Die Ausbreitung von Tau kann in vivo auch mittels Tau-PET dargestellt werden. Es folgt die Neurodegeneration, bei der die zerebrale Atrophie mittels MRT-Untersuchungen und die neuronale Funktionsstörung durch FDG-PET-Untersuchungen nachgewiesen werden können. Auf die pathophysiologischen Veränderungen der Alzheimer-Krankheit folgen zeitlich versetzt die klinischen Symptome. Mit dem Nachweis von Neurodegeneration sind die Patienten häufig symptomatisch und haben das Prodromalstadium (das MCI-Stadium) oder das Demenzstadium der AD bereits erreicht. Dieses Modell verdeutlicht auch, dass die klinischen Funktionseinschränkungen als Kontinuum $\mathrm{zu}$ verstehen sind, mit fließenden Übergängen zwischen dem kognitiv normalen Zustand über das MCI-Stadium bis hin zur Demenz. Bedeutsam ist, dass es sich bei der beschriebenen Chronologie um ein vereinfachtes Modell der Amyloid-Hypothese handelt. Das vorzeitige Ende einiger amyloidbasierter klinischer Studien spricht grundsätzlich nicht gegen die Amyloid-Hypothese, sondern unterstreicht die Notwendigkeit primärpräventiv angelegter krankheitsmodifizierender klinischer Studien.

\section{Früherkennung der Alzheimer- Krankheit und Demenzprädikti- on mit Biomarkern}

Untersuchungen haben gezeigt, dass die klinische Diagnose der Alzheimer-Demenz in nur $70-80 \%$ der Fälle mit der Alzheimer-Pathologie in postmortem Untersuchungen korreliert [3, 29]. Eine Metaanalyse zum Einsatz von AmyloidPET-Untersuchungen bei Patienten mit klinischer Demenzdiagnose zeigte, dass $12 \%$ aller Patienten mit einer AlzheimerDemenz keine Amyloid-Pathologie im PET aufwiesen [42]. Diese diagnostische Unsicherheit ist unter anderem darin begründet, dass die Symptome der AD nicht vollständig spezifisch sind und es insbesondere mit steigendem Alter Überlappungen mit anderen neurodegenerativen bzw. vaskulären Pathologien gibt, die ähnliche Symptome bedingen können. Bei MCI zeigen etwa 50-60\% aller Patienten keine biomarkerbasierten bzw. neuropathologischen Korrelate der $\mathrm{AD}[47,53]$.

Die mangelnde Spezifität klinischer Symptome für die AD-Pathologie kann durch Biomarkeruntersuchungen für die Alzheimer-Krankheit kompensiert werden. Im MCI-Stadium ist durch Bestimmung der Biomarker ein Ausschluss bzw. Nachweis der AD-Pathologie möglich [21, 48]. Basierend auf der derzeit umfassendsten Metaanalyse zur biomarkerbasierten AD-Früherkennung können für MCI-Patienten differenzierte Aussagen zum Krankheitsverlauf und der Wahrscheinlichkeit für eine Demenz getroffen werden ([52]; • Tab. 3). Die Risikoschätzungen basieren auf der $\mathrm{A} / \mathrm{N}$ Einteilung $[25,52]$. Die Grenzen der Da- 


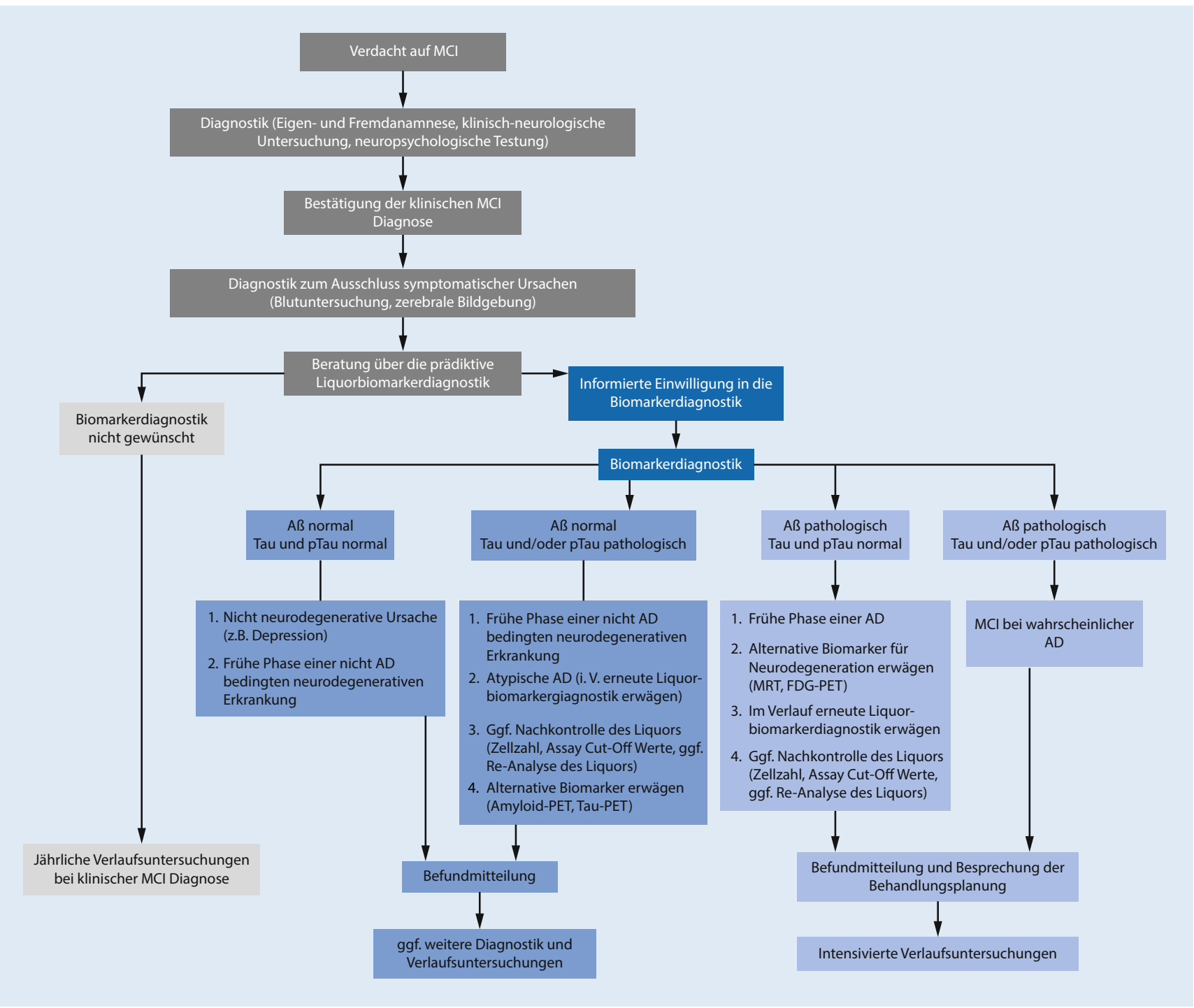

Abb. $1 \Delta$ Entscheidungshilfe für die diagnostische Abklärung und prädiktive Diagnostik bei „"mild cognitive impairment" (MCI). pTau phosphoryliertes Tau, tTau gesamtes Tau, MRT Magnetresonanztomographie, PET Positronenemissionstomographie, FDG Fluordesoxyglucose. (Mod. nach [21])

tengrundlage für die biomarkerbasierte Demenzprädiktion sind derzeit unter anderem darin begründet, dass es noch keine zentrumsübergreifenden Methodenstandards und Normwerte für ADBiomarker gibt und bisher keine individuellen Patientencharakteristika (Alter, Geschlecht, komorbide Erkrankungen etc.) in die Prädiktionsmodelle integriert wurden [52]. Kürzlich entwickelte eine niederländische Arbeitsgruppe ein biomarkerbasiertes Prädiktionsmodell aus dem Alzheimer's Biomarkers in Daily Practice (ABIDE) Projekt und legte einen wichtigen Grundstein für die individualisierte Risikoprofilanalyse von Personen mit MCI $[36,37]$. Als Grundlage dienten
Daten von über 2600 MCI-Patienten aus 4 großen Kohorten (European Medical Information Framework for Alzheimer's disease [EMIF], Alzheimer's Disease Neuroimaging Initiative [ADNI], Amsterdam Dementia Cohort [ADC] und Swedish BioFINDER studies). Es wurden insgesamt 4 verschiedene Prädiktionsmodelle untersucht, die die demographischen Daten Geschlecht, Alter und Mini-Mental-Status-Test (MMST), die individuellen Liquorbiomarkerdaten, MRT-basierte hippokampale Volumina sowie das A/T/N-Modell berücksichtigten und Übergangsraten in eine Demenz innerhalb von 1, 3 und 5 Jahren berechneten. Die Autoren haben bei der Validierung des biomarkerbasierten Prädiktionsmodells unter anderem verschiedene Assays für Liquoranalysen und die verschiedene Software für die MRT-basierte Bestimmung der hippokampalen Volumina berücksichtigt. Die Untersuchungen zeigten, dass das A/T/N-Modell die präziseste Prädiktion hinsichtlich des Demenzrisikos liefert [36]. Da die prädiktive Liquordiagnostik mittlerweile eine gängige klinische Praxis ist, gewinnt die Qualitätskontrolle zunehmend an Bedeutung. Durch automatisierte Assay-Durchführung kann die methodische Zuverlässigkeit optimiert werden. In diesem Zusammenhang hat eine der größten europäischen 
Forschungsverbünde, mit über 18 teilnehmenden Ländern, das BIOMARKAPD-Projekt, maßgeblich zur Standardisierung und Optimierung von Probengewinnung, Probenlagerung, Probentransport sowie Probenbearbeitung beigetragen. Der Einsatz von AmyloidPET als Früherkennungsuntersuchung der $\mathrm{AD}$ und für die individuelle Prädiktion einer Alzheimer-Demenz in Prädemenzstadien wird derzeit intensiv beforscht [35, 56]. Aus Studien ist bekannt, dass die Anwendung von Amyloid-PET, ähnlich wie der Einsatz von Liquorbiomarkern, im MCI- und Demenzstadium die Patientenversorgung verbessern kann (Diagnoserevision und Anpassung des klinischen Managements; $[46,55])$. Aufgrund noch fehlender pharmakologischer Ansätze im prodromalen AD-Stadium wirken sich die Änderungen des klinischen Managements bei Amyloidpositivität für MCI-Patienten geringer aus, als die Änderungen der Krankheitsprognose bei Amyloidpositivität. Kriterienansätze für die klinische Anwendung der Amyloid-PET-Diagnostik bei MCI- und Demenzpatienten wurden von einer US-amerikanischen Expertengruppe entwickelt (Amyloid Imaging Task Force, the Society of Nuclear Medicine and Molecular Imaging [SNMMI] und Alzheimer Association; [27]). Der Einsatz der Amyloid-PET im Rahmen einer AD-Früherkennungsdiagnostik erfolgt derzeit primär im Rahmen von (klinischen) Studien. Dies ist unter anderem darin begründet, dass die prädiktive und prognostische Wertigkeit weiter untersucht werden müssen $[35,56]$. In Deutschland wird derzeit die Kostenübernahme durch die gesetzlichen Krankenkassen für Amyloid-PET in der Regel nicht gewährt.

Zusammenfassend sind die Grenzen der Datengrundlage für die biomarkerbasierte Demenzprädiktion derzeit unter anderem darin begründet, dass es noch keine zentrumsübergreifenden Methodenstandards und Normwerte für $\mathrm{AD}$-Biomarker gibt, auch müssen oben genannte Prädiktionsmodelle in prospektiven Studien weiter validiert werden, ehe sie in der klinischen Praxis eingesetzt werden können.
Auswirkungen der Bestimmung von Biomarkern auf das klinische Management und das psychische Wohlbefinden

Die Datenlage zu den Effekten der biomarkerbasierten Früherkennung der AD auf das klinische Management ist begrenzt. Die Studienlage zeigt, dass bei MCI und Demenz die Durchführung von Biomarkeruntersuchungen in 7-60\% der Fälle zu einer Diagnoseänderung und somit Anpassung der Behandlungsplanung führt [9, 28, 40, 46, 54]. Kürzlich publizierte Arbeiten der US-amerikanischen Amyloid-PET-Studie Imaging Dementia - Evidence for Amyloid Scanning (IDEAS) und des eingangs erwähnten niederländischen ABIDE-Projektes belegten Änderungen der Therapiestrategien (Umstellung der medikamentösen Therapie, intensivierte Beratungsangebote, optionaler Einschluss in klinische Studien) in Abhängigkeit der Resultate der Amyloid-PET-Untersuchungen [46, 54]. Die Autoren der Studien kommen zu dem Schluss, dass der Einsatz von Biomarkern für Ärzte einen Zugewinn an Informationen und an Sicherheit in der diagnostischen Zuordnung bietet.

Aufgrund derzeit fehlender substanziell wirksamer Behandlungsoptionen, hat die Früherkennung der AD in den Prädemenzstadien keine unmittelbare Auswirkung auf die leitliniengerechte medikamentöse Therapie [21, 48]. Die in den S3-Leitlinien empfohlenen symptomatischen Therapien mit Acetylcholinsterasehemmern (Donepezil, Rivastigmin, Galantamin) und Modulatoren des glutamatergen Systems (Memantin; [10] sind ausschließlich für das Demenzstadium zugelassen. Die Datenlage zu gesundheitsökonomischen Folgen der Liquorbiomarkeruntersuchung ist sehr limitiert. Nur eine Arbeit adressierte die möglichen Kosteneffekte von Liquoruntersuchungen, bei denen unter anderem die Auswirkungen auf die Verordnung von Donepezil bei Patienten mit MCI („off-label“) bzw. klinischer Demenzdiagnose untersucht wurden [50].

Die Auswirkungen auf das psychische Wohlbefinden nach Mitteilung der Biomarkerergebnisse sind in verschie- denen Patientenkollektiven (subjektive kognitive Störung, MCI und leichte Demenz) untersucht worden [16, 33, 41, 51]. Grundsätzlich zeigen diese ersten Studien, dass die Mitteilung von Biomarkerergebnissen bei Patienten und deren Angehörigen, auch bei nachgewiesener Alzheimer-Pathologie, in der Regel nicht $\mathrm{zu}$ klinisch ausgeprägten psychischen Belastungsreaktionen führt.

Bei diesen Ergebnissen muss berücksichtigt werden, dass im Kontext klinischer Studien eine ausführliche Beratung der Studienprobanden über die Biomarkerdiagnostik vorausging. Darüber hinaus gibt es bei der Beratung von MCIPatienten und Demenzpatienten relevante inhaltliche Unterschiede. Während im Demenzstadium die AD-Biomarker genutzt werden, um eine Alzheimer-Pathologie zu bestätigen bzw. auszuschließen, ermöglichen sie im Prädemenzstadium neben dem Nachweis der Alzheimer-Pathologie auch eine Prognose zum Krankheitsverlauf und eine Demenzprädiktion. Die Demenzprädiktion kann wiederum erhebliche Auswirkungen auf die psychosoziale Situation und die weitere Lebensplanung der Betroffenen und Angehörigen haben. Differenzierte Untersuchungen zu den Auswirkungen der biomarkerbasierten Früherkennung der $\mathrm{AD}$ und der Demenzprädiktion in Prädemenzstadien auf Patienten und Angehörige werden zurzeit durchgeführt.

\section{Empfehlungen für den Einsatz von Biomarkern bei $\mathrm{MCl}-$ Patienten}

Obwohl die Liquorbiomarkeruntersuchung im MCI-Stadium in Deutschland eine gängige Praxis ist, gibt es für deren Einsatz zur Früherkennung der AD und zur Demenzprädiktion keine Empfehlungen oder Leitlinien. Die S3-Leitlinie Demenzen bietet Empfehlungen zur differenzialdiagnostischen Anwendung der Liquoruntersuchung bei Demenz [10], jedoch wird (noch) keine explizite Handlungsanweisung zur biomarkerbasierten Früherkennung der $\mathrm{AD}$ und der Demenzprädiktion beim MCI gegeben.

Die Autoren der europäischen (BIOMARK-APD) und US-amerikanischen (AA) Konsensuspublikationen legen 
Tab.4 Vorgehensempfehlungen für das Beratungsgespräch über die Biomarkerdiagnostik der Alzheimer-Krankheit. (Mod. nach [20,21])

Psychoedukation zur Dynamik des Krankheitsmodells und der Symptomentwicklung

Erläuterung der allgemeinen Prognose basierend auf den klinischen MCI-Kriterien

Aufklärung über die Möglichkeit der Früherkennung der AD bereits im MCI-Stadium durch Liquorbiomarker

Informationen zu Lumbalpunktion

Erläuterung der biomarkerbasierten Demenzprädiktion anhand von 3 verschiedenen Szenarien in Bezug auf das Demenzrisiko

Informationen zu protektivem Lebensstil (körperliche, geistige und soziale Aktivität, mediterrane Ernährung, Einstellung der kardiovaskulären Risikofaktoren)

Informationen zu kognitiven Interventionen (kognitive Stimulation) und zu Ergotherapie

Symptomatische pharmakologische Behandlung bei Progression zur Demenz ansprechen

Die wesentlichen Information durch den Patienten und Angehörige wiederholen lassen

Patienten und Angehörige anregen, das Für und Wider der AD-Früherkennung und der Demenzprädiktion zu besprechen (z. B. im Familienverbund, mit nahestehenden Personen, dem Hausarzt etc.)

Ausreichend Zeit bis zur Entscheidung für/gegen die Biomarkerdiagnostik einräumen

Empfehlungen zum Einsatz von Biomarkern bei MCI vor, die im Folgenden zusammengefasst werden [21, 48].

Grundsätzlich sollten vor der Biomarkeruntersuchung das MCI-Syndrom klinisch bestätigt und durch die unten aufgeführten diagnostischen Maßnahmen potenziell behandelbare Ursachen erkannt werden (• Tab. 1; $[1,10,21$, 30]). Das MCI-Syndrom kann anhand des klinischen Bildes nach ausführlicher Eigen- und Fremdanamnese und durch Ergänzung der neuropsychologischen Testung festgestellt werden. Als nächste Maßnahmen sollten sich eine Blutuntersuchung und die zerebrale Bildgebung (Magnetresonanztomographie [MRT] oder Computertomographie [CT]) anschließen, um mit diesen Verfahren diagnostizierbare Ursachen des MCI auszuschließen (『Tab. 1). Mögliche Ursachen können psychische Erkrankungen (z.B. depressive Störung, Angststörung, Schlafstörung), Arzneimittelnebenwirkungen oder -interaktionen, internistische oder neurologische bzw. neurodegenerative Erkrankungen sein. Von der europäischen BIOMARK-APD-Projektgruppe wurde eine Entscheidungshilfe für das klinisch-diagnostische Vorgehen mit und ohne Biomarkeruntersuchung bei MCI entwickelt (• Abb. 1; [21]).

Nach Bestätigung der MCI-Diagnose und Ausschluss spezifischer Ursachen sollte im Anschluss eine Früherkennung der Alzheimer-Krankheit und der damit verknüpften Möglichkeit der Demenzprädiktion erwogen werden [21, 48]. Hierbei betonen beide Konsensuspublikationen die informierte Einwilligung des Patienten in die Biomarkerdiagnostik nach umfassender Beratung über die Möglichkeiten und Risiken der Früherkennung der Alzheimer-Krankheit und der Demenzprädiktion als entscheidende Voraussetzung für die Untersuchungen. Das Beratungsgespräch dient dazu, MCI-Patienten und ihre Angehörigen anzuregen, sich mit den Themen Biomarkeruntersuchung und Demenzprädiktion sowie dem individuellen Für und Wider auseinanderzusetzen. Inhalte des Beratungsgesprächs sollten Informationen zum Krankheitsmodell und zur Symptomentwicklung sowie zu (präventiven) Ansätzen, u. a. durch Lebensstilmodifikationen, sein (๑ Tab. 4; [18]).

Kernthemen des Beratungsgespräches sollten ferner die Möglichkeit der frühen Feststellung einer AD bereits im MCIStadium und die Erläuterung möglicher Biomarkerresultate in Bezug auf das Demenzrisiko sein. Insbesondere sollten die Patienten und Angehörigen darauf aufmerksam gemacht werden, dass die Früherkennung der AD im MCI-Stadium eine Prognose zum Krankheitsverlauf und zum Demenzrisiko ermöglicht, jedoch keine Demenzdiagnose darstellt.

Die Prädiktion einer noch nicht behandelbaren Erkrankung, wie der Alzhei-
mer-Demenz, kann zu erheblichen Belastungen führen, daher sollte im Beratungsgespräch darauf hingewiesen werden, dass nach der Befundmitteilung psychologische Begleitreaktionen, Veränderungen im sozialen und beruflichen Umfeld, neue sozialrechtliche Herausforderungen und mögliche Benachteiligungen bei Versicherungen auftreten können [2, 6, 20, 21, 51].

Grundsätzlich ist aus der Literatur und den Erfahrungen aus Gedächtnisambulanzen bekannt, dass viele Patienten, trotz derzeit fehlender Optionen eine mögliche Demenz substanziell aufzuhalten, über die $\mathrm{AD}$ und ihr individuelles Demenzrisiko informiert werden möchten, um ihre weitere Lebensplanung danach auszurichten und durch gezielte präventive Therapieansätze eine Verlangsamung der Krankheitsprogression $\mathrm{zu}$ erzielen [13, 17, 34, 51].

Grundsätzlich kann basierend auf den klinischen MCI-Diagnosekriterien [1] und bei unbekanntem Biomarkerstatus eine allgemeine Prognose über den Krankheitsverlauf mitgeteilt werden [38]. Hierbei entspricht das Risiko für Personen mit MCI, innerhalb von 3 Jahren eine Alzheimer-Demenz zu entwickeln, ca. 33\%. [38]. Da etwa 50-60\% aller MCI-Patienten keine AlzheimerPathologie aufzeigen $[47,53]$ und somit ein geringeres Risiko für den Übergang in eine Alzheimer-Demenz haben als MCI-Patienten mit einer AlzheimerPathologie [7, 52], ist ohne eine Biomarkeruntersuchung eine differenzierte Prognose im Hinblick auf das Demenzrisiko nicht möglich. Die biomarkerbasierte Feststellung der $\mathrm{AD}$ ermöglicht es, MCI-Patienten genauere Prognosen zum Krankheitsverlauf mitzuteilen [21, 34, 51]. Je nach Konstellation des Biomarkerprofils können aktuell drei verschiedene Szenarien in Bezug auf das Demenzrisiko unterschieden werden.

Beim Vorliegen einer AD (Amyloidund Tau-Pathologie) liegt die Wahrscheinlichkeit, innerhalb von 5 Jahren nach der MCI-Diagnosestellung eine Alzheimer-Demenz zu entwickeln, bei fast $90 \%$ [52]. Sind nur eine Amyloidoder eine Tau-Pathologie vorhanden, liegt das Übergangsrisiko innerhalb von 5 Jahren nach der MCI-Diagnosestel- 
Tab. 5 Vorgehensempfehlungen für die Befundmitteilung und die klinischen Verlaufskontrollen bei MCl-Patienten mit Alzheimer-Krankheit. (Mod. nach [20, 21])

Vorgehensempfehlung bei Bestätigung der Alzheimer-Krankheit in der Biomarkerdiagnostik

Psychoedukation zur Dynamik des Krankheitsmodells und der Symptomentwicklung

Aufklären über mögliche Depressivität, Ängste, Schlafstörungen und Suizidalität

Informationen zu kognitiven Interventionen (kognitive Stimulation) und zu Ergotherapie

Informationen zu protektivem Lebensstil (körperliche, geistige und soziale Aktivität, mediterrane Ernährung, Einstellung der kardiovaskulären Risikofaktoren)

Frühzeitige symptomatische pharmakologische Behandlung bei Progression zur Demenz Kurzfristige Verlaufstermine anbieten

Auf jederzeit mögliche Kontaktaufnahme durch den Patienten oder die Angehörigen hinweisen und sicherstellen, dass die Kontaktinformation vorhanden ist (insbesondere bei Suizidalität)

Die wesentlichen Information durch den Patienten und Angehörige wiederholen lassen

Klinische Verlaufskontrollen: nach 1 bis 4 Wochen, 6 Monaten und 12 Monaten

lung zwischen 45-50\%. Bei unauffälligen Biomarkern liegt das Risiko für eine Alzheimer-Demenz bei circa $10 \%$.

Wird vom Patienten eine Biomarkeruntersuchung gewünscht, schließt sich nach der Lumbalpunktion eine Befundmitteilung an, die, soweit möglich, in Anwesenheit eines Angehörigen oder einer nahestehenden Person durchgeführt werden sollte. Grundsätzlich wird empfohlen, die Befundmitteilung in einer einfach verständlichen Sprache zu vermitteln, ausreichend Zeit für Rückfragen einzuplanen und auf mögliche psychische Belastungsreaktionen zu achten [20, 34]. Zur Vermittlung des Demenzrisikos sind visuelle Hilfsmitteln wie Grafiken, Balkendiagramme oder Piktogramme nützlich, die bisher nur für Studienzwecke entwickelt worden sind [15, 20, 34]. Hilfreich ist zudem die Anwendung der Teach-back-Methode, bei der die Patienten und Angehörigen gebeten werden, die wesentlichen Inhalte der Befundmitteilung in eigenen Worten wiederzugeben. Bei der Befundbesprechung sollten erneut die präventiven Therapieansätze erläutert und die Patienten und Angehörigen über mögliche psychische Begleitreaktionen aufgeklärt werden (• Tab. 5; [20, 21, 48]). Wird eine $\mathrm{AD}$ nachgewiesen und liegt damit ein erhöhtes Demenzrisiko vor, sollte ein Behandlungsplan mit regelmäßigen klinischen Verlaufskontrollen einschließlich der Behandlung komorbider Erkrankungen aufgestellt werden. Falls ein Fortschreiten zur Demenz stattfindet, wird der frühestmögliche Beginn

\section{Korrespondenzadresse}

Dr. Ayda Rostamzadeh

Klinik für Psychiatrie und Psychotherapie, Uniklinik Köln, Medizinische Fakultät Köln, Deutschland

ayda.rostamzadeh@uk-koeln.de

Funding. Open Access funding provided by Projekt DEAL.

\section{Einhaltung ethischer Richtlinien}

Interessenkonflikt. A. Rostamzadeh und F. Jessen geben an, dass kein Interessenkonflikt besteht.

Für diesen Beitrag wurden von den Autoren keine Studien an Menschen oder Tieren durchgeführt. Für die aufgeführten Studien gelten die jeweils dort angegebenen ethischen Richtlinien.

Therapie empfohlen.

\section{Fazit für die Praxis}

Die Anwendung der biomarkerbasierten Früherkennung der AD und Demenzprädiktion bei MCl-Patienten wird in der klinischen Praxis immer häufiger angefragt. Aufgrund fehlender nationaler Leitlinienempfehlungen und der komplexen Interpretation von Biomarkerprofilen werden besondere Herausforderungen an Patienten, Angehörige und Ärzte gestellt. Grundsätzlich wird empfohlen, vor der Durchführung von Biomarkeruntersuchungen auf die Möglichkeiten und Grenzen einer prädiktiven Diagnostik hinzuweisen und Patienten und Angehörige im Sinne eines „informed-consent" und "Shareddecision-making"-Prozesses zu begleiten. Durch die Früherkennung der AD können individuelle Prognosen zum Krankheitsverlauf und zur Prädiktion der Alzheimer-Demenz gemacht werden. $\mathrm{MCl}$-Patienten mit AD haben ein fast $\mathbf{9 0 \% i g e s ~ R i s i k o ~ i n n e r h a l b ~ v o n ~}$ 5 Jahren eine Alzheimer-Demenz zu entwickeln. Da die AD nicht heilbar ist, können diese Prognoseabschätzungen Folgen für die Lebensplanung und das psychische Wohlbefinden der Betroffenen haben, weshalb regelmäßige Verlaufskontrollen mit Angeboten zu Präventions- und frühen Therapiemaßnahmen empfohlen werden.
Open Access. Dieser Artikel wird unter der Creative Commons Namensnennung 4.0 International Lizenz veröffentlicht, welche die Nutzung, Vervielfältigung, Bearbeitung, Verbreitung und Wiedergabe in jegliursprünglichen Autor(en) und die Quelle ordnungsgemäß nennen, einen Link zur Creative Commons Lizenz beifügen und angeben, ob Änderungen vorgenommen wurden.

Die in diesem Artikel enthaltenen Bilder und sonstiges Drittmaterial unterliegen ebenfalls der genannten Creative Commons Lizenz, sofern sich aus der Abbildungslegende nichts anderes ergibt. Sofern das betreffende Material nicht unter der genannten Creative Commons Lizenz steht und die betreffende Handlung nicht nach gesetzlichen Vorschriften erlaubt ist, ist für die oben aufgeführten Weiterverwendungen des Materials die Einwilligung des jeweiligen Rechteinhabers einzuholen.

Weitere Details zur Lizenz entnehmen Sie bitte der Lizenzinformation auf http://creativecommons.org/ licenses/by/4.0/deed.de.

\section{Literatur}

1. Albert MS, DeKosky ST, Dickson D et al (2011) The diagnosis of mild cognitive impairment due to Alzheimer's disease: recommendations Association workgroups on diagnostic guidelines for Alzheimer's disease. Alzheimers Dement 7(3):270-279. https://doi.org/10.1016/j.jalz.2011. 03.008

2. Banningh LJW, Vernooij-Dassen M, Rikkert MO, Teunisse JP (2008) Mild cognitive impairment: coping with an uncertain label. Int J Geriatr Psychiatry 23:148-154. https://doi.org/10.1002/ gps. 1855

3. Beach TG, Monsell SE, Phillips LE, Kukull W (2012) Accuracy of the clinical diagnosis of Alzheimer disease at National Institute on Aging Alzheimer Disease Centers, 2005-2010. J Neuropathol Exp Neurol. https://doi.org/10. 1097/NEN.0b013e31824b211b chem Medium und Format erlaubt, sofern Sie den/die from the National Institute on Aging-Alzheimer's 
4. Blennow K, de Leon MJ, Zetterberg H (2006) Alzheimer's disease. Lancet 368:387-403. https:// doi.org/10.1016/S0140-6736(06)69113-7

5. BraakH,BraakE(1991)Neuropathologicalstageing of Alzheimer-related changes. Acta Neuropathol 82:239-259. https://doi.org/10.1007/BF00308809

6. Bronner K, Perneczky R, McCabe R et al (2016) Which medical and social decision topics are important after early diagnosis of Alzheimer's Disease from the perspectives of people with Alzheimer's Disease, spouses and professionals? BMC Res Notes 9:149. https://doi.org/10.1186/ s13104-016-1960-3

7. Buchhave $P$, Minthon L, Zetterberg $\mathrm{H}$ et al (2012) Cerebrospinal fluid levels of $\beta$-amyloid 1-42, but not of tau, are fully changed already 5 to 10 years before the onset of Alzheimer dementia. Arch Gen Psychiatry 69:98-106. https://doi.org/10.1001/ archgenpsychiatry.2011.155

8. Chhatwal JP, Schultz AP, Marshall GA et al (2016) Temporal T807 binding correlates with CSF tau and phospho-tau in normal elderly. Neurology 87:920-926. https://doi.org/10.1212/ WNL.0000000000003050

9. Cognat E, Troussière A-C, Wallon D et al (2017) Clinical impact of cerebrospinal fluid biomarkers in mild cognitive impairment diagnosis. Alzheimers Dement. https://doi.org/10.1016/j.jalz.2017.06. 060

10. Deuschl G, Maier W (2016) S3-Leitlinie Demenzen. Deutsche Gesellschaft für Neurologie

11. Fagan AM, Mintun MA, Mach RHetal (2006) Inverse relation between in vivo amyloid imaging load and cerebrospinal fluid $A \beta_{42}$ in humans. Ann Neurol 59:512-519. https://doi.org/10.1002/ana.20730

12. Glodzik-Sobanska L, Rusinek H, Mosconi L et al (2005) The role of quantitative structural imaging in the early diagnosis of Alzheimer's disease. Neuroimaging Clin N Am. https://doi.org/10.1016/ j.nic.2005.09.004

13. Gooding $H C$, Linnenbringer EL, Burack J et al (2006) Genetic susceptibility testing for Alzheimer disease: motivation to obtain information and control as precursors to coping with increased risk. Patient Educ Couns 64:259-267. https://doi.org/ 10.1016/j.pec.2006.03.002

14. Gordon BA, Friedrichsen K, Brier M et al (2016) The relationship between cerebrospinal fluid markers of Alzheimer pathology and positron emission tomography tau imaging. Brain 139:2249-2260. https://doi.org/10.1093/brain/aww139

15. Green RC, Roberts JS, Cupples LA et al (2009) Disclosure of APOE genotype for risk of Alzheimer's disease. N Engl J Med 361:245-254. https://doi. org/10.1056/NEJMoa0809578

16. Grill JD, Cox CG, Kremen S et al (2017) Patient and caregiver reactions to clinical amyloid imaging. Alzheimers Dement 13:924-932. https://doi.org/ 10.1016/j.jalz.2017.01.001

17. Guan Y, Roter DL, Erby LH et al (2017) Disclosing genetic risk of Alzheimer's disease to cognitively impaired patients and visit companions: findings from the REVEAL study. Patient Educ Couns 100:927-935. https://doi.org/10.1016/j.pec.2016. 12.005

18. World Health Organization (2019) Risk reduction of cognitive decline and dementia (Evidence profile: Physical activity and cognitive decline or dementia)

19. Handels RLH, Vos SJB, Kramberger MG et al (2017) Predicting progression to dementia in persons with mild cognitive impairment using cerebrospinal fluid markers. Alzheimers Dement
13:903-912. https://doi.org/10.1016/j.jalz.2016. 12.015

20. Harkins K, Sankar P, Sperling R et al (2015) Development of a process to disclose amyloid imaging results to cognitively normal older adult research participants. Alzheimers Res Ther 7:26. https://doi.org/10.1186/s13195-015-0112-7

21. Herukka S-K, Simonsen AH, Andreasen N et al (2017) Recommendations for cerebrospinal fluid Alzheimer's disease biomarkers in the diagnostic evaluation of mild cognitive impairment. Alzheimers Dement 13(3):285-295. https://doi.org/10. 1016/j.jalz.2016.09.009

22. Hu C, Yu D, Sun X et al (2017) The prevalence and progression of mild cognitive impairment among clinic and community populations: a systematic review and meta-analysis. Int Psychogeriatr 29:1595-1608. https://doi.org/10. 1017/S1041610217000473

23. Jack CR, Bennett DA, Blennow $\mathrm{K}$ et al (2016) $\mathrm{A} / \mathrm{T} / \mathrm{N}$ : an unbiased descriptive classification scheme for Alzheimer disease biomarkers. Neurology 87:539-547. https://doi.org/10.1212/WNL. 0000000000002923

24. Jack CR, Bennett DA, Blennow $\mathrm{K}$ et al (2018) NIA-AA research framework: toward a biological definition of Alzheimer's disease. Alzheimers Dement 14:535-562. https://doi.org/10.1016/j. jalz.2018.02.018

25. Jack CR, Knopman DS, Jagust WJ et al (2013) Tracking pathophysiological processes in Alzheimer's disease: an updated hypothetical model of dynamic biomarkers. Lancet Neurol 12:207-216. https://doi.org/10.1016/S1474-4422(12)70291-0

26. Jansen WJ, Ossenkoppele R, Knol DL et al (2015) Prevalence of cerebral amyloid pathology in persons without dementia: a meta-analysis. JAMA. https://doi.org/10.1001/jama.2015.4668

27. Johnson KA, Minoshima S, Bohnen NI, Donohoe KJ, Foster NL, Herscovitch P, Karlawish JH, Rowe CC, Carrillo MC, Hartley DM, Hedrick S, Mitchell K, Pappas V, Thies WH (2013) Appropriate use criteria for amyloid PET: a report of the Amyloid Imaging Task Force, the Society of Nuclear Medicine and Molecular Imaging, and the Alzheimer's Association. AlzheimersDement 9:E1-E16.https:// doi.org/10.1016/j.jalz.2013.01.002

28. Kester MI, Boelaarts L, Bouwman FH et al (2010) Diagnostic impact of CSF biomarkers in a local hospital memory clinic. Dement Geriatr Cogn Disord. https://doi.org/10.1159/000313534

29. Knopman DS, Dekosky ST, Cummings JL et al (2001) Practice parameter: diagnosis of dementia (an evidence-based review). Report of the Quality Standards Subcommittee of the American Academy of Neurology. Neurology 56(9):1143-1153. https://doi.org/10.1212/wnl.56.9.1143

30. Knopman DS, Petersen RC (2014) Mild cognitive impairment and mild dementia: a clinical perspective. Mayo Clin Proc 89:1452-1459. https://doi. org/10.1016/j.mayocp.2014.06.019.Mild

31. Lewczuk P, Matzen A, Blennow K et al (2016) Cerebrospinal fluid $A \beta 42 / 40$ corresponds better than $A \beta 42$ to amyloid PET in Alzheimer's disease. J Alzheimers Dis 55:813-822. https://doi.org/10. 3233/JAD-160722

32. Lewczuk P, Riederer P, O'Bryant SE et al (2017) Cerebrospinal fluid and blood biomarkers for neurodegenerative dementias: an update of the consensus of the Task Force on Biological Markers in Psychiatry of the World Federation of Societies of Biological Psychiatry. World J Biol Psychiatry. https://doi.org/10.1080/15622975.2017.1375556
33. Lim YY, Maruff P, Getter C, Snyder PJ (2016) Disclosure of positron emission tomography amyloid imaging results: a preliminary study of safety and tolerability. Alzheimers Dement 12:454-458. https://doi.org/10.1016/j.jalz.2015. 09.005

34. Lingler JH, Butters MA, Gentry AL et al (2016) Development of a standardized approach to disclosing amyloid imaging research results in mild cognitive impairment. J Alzheimers Dis 52:17-24. https://doi.org/10.3233/JAD-150985

35. Martínez G, Vernooij RWM, Fuentes Padilla P et al (2017) 18F PET with flutemetamol for the early diagnosis of Alzheimer's disease dementia and other dementias in people with mild cognitive impairment (MCl). Cochrane Database Syst Rev. https://doi.org/10.1002/14651858.CD012884

36. van Maurik IS, Vos SJ, Bos I et al (2019) Biomarkerbased prognosis for people with mild cognitive impairment (ABIDE): a modelling study. Lancet Neurol 4422:1-11. https://doi.org/10.1016/ S1474-4422(19)30283-2

37. van Maurik IS, Zwan MD, Tijms BM et al (2017) Interpreting biomarker results in individual patients with mild cognitive impairment in the Alzheimer's Biomarkers in Daily Practice (ABIDE) project. JAMA Neurol. https://doi.org/10.1001/ jamaneurol.2017.2712

38. Mitchell AJ, Shiri-Feshki M (2009) Rate of progression of mild cognitive impairment to dementia-meta-analysis of 41 robust inception cohort studies. Acta Psychiatr Scand 119:252-265. https://doi.org/10.1111/j.1600-0447.2008.01326.

39. Mosconi L, Tsui WH, De Santi S et al (2005) Reduced hippocampal metabolism in $\mathrm{MCl}$ and $\mathrm{AD}$. automated FDG-PET image analysis. Neurology https://doi.org/10.1212/01.WNL.0000163856. 13524.08

40. Mouton-Liger $F$, Wallon $D$, Troussière $A C$ et al (2014) Impact of cerebro-spinal fluid biomarkers of Alzheimer's disease in clinical practice: a multicentric study. J Neurol. https://doi.org/10. 1007/s00415-013-7160-3

41. Mozersky J, Sankar P, Harkins K et al (2018) Comprehension of an elevated amyloid positron emission tomography biomarker result by cognitively normal older adults. JAMA Neurol. https:// doi.org/10.1001/jamaneurol.2017.2954

42. Ossenkoppele R, Jansen WJ, Rabinovici GD et al (2015) Prevalence of amyloid PET positivity in dementia syndromes: a meta-analysis. JAMA https://doi.org/10.1001/jama.2015.4669

43. Petersen RC (2004) Mild cognitive impairment as a clinical entity and treatment target. Arch Neurol 62:1160-1163. https://doi.org/10.1001/archneur. 62.7.1160 (discussion 1167)

44. Petersen RC, Caracciolo B, Brayne C et al (2014) Mild cognitive impairment: a concept in evolution J Intern Med 275:214-228. https://doi.org/10. 1111/joim.12190

45. Petersen RC, Lopez O, Armstrong MJ et al (2018) Practice guideline update summary:mild cognitive impairment. Neurology 90(3):126-135. https:// doi.org/10.1212/WNL.0000000000004826

46. Rabinovici GD, Gatsonis C, Apgar C et al (2019) Association of amyloid positron emission tomography with subsequent change in clinical management among medicare beneficiaries with mild cognitive impairment or dementia. JAMA. https://doi.org/10.1001/jama.2019.2000

47. Schneider JA, Arvanitakis Z, Leurgans SE, Bennett DA (2009) The neuropathology of probable 
Alzheimer disease and mild cognitive impairment Ann Neurol. https://doi.org/10.1002/ana.21706

48. Shaw LM, Arias J, Blennow K et al (2018) Appropriate use criteria for lumbar puncture and cerebrospinal fluid testing in the diagnosis of Alzheimer's disease. Alzheimers Dement. https:// doi.org/10.1016/j.jalz.2018.07.220

49. Smith R, Wibom M, Pawlik Detal (2018) Correlation of in vivo [18F]flortaucipir with postmortem Alzheimer disease tau pathology. JAMA Neurol. https://doi.org/10.1001/jamaneurol.2018.3692

50. Valcárcel-Nazco C, Perestelo-Pérez L, Molinuevo JL, Mar J, Castilla I, Serrano-Aguilar P (2014) Costeffectiveness of the use of biomarkers in cerebrospinal fluid for Alzheimer's disease. J Alzheimers Dis 42(3):777-788. https://doi.org/10.3233/JAD132216

51. Vanderschaeghe G, Schaeverbeke J, Vandenberghe R, Dierickx K (2017) Amnestic MCl patients' perspectives toward disclosure of amyloid PET results in a research context. Neuroethics 10:281-297. https://doi.org/10.1007/s12152-017-9313-z

52. Vos SJB, Verhey F, Frölich L et al (2015) Prevalence and prognosis of Alzheimer's disease at the mild cognitive impairment stage. Brain 138:1327-1338. https://doi.org/10.1093/brain/awv029

53. Wagner M, Wolf S, Reischies FM et al (2012) Biomarker validation of a cued recall memory deficit in prodromal Alzheimer disease. Neurology 78(6):379-386. https://doi.org/10.1212/WNL. 0b013e318245f447

54. De Wilde A, Van Der Flier WM, Pelkmans W et al (2018) Association of amyloid positron emission tomography with changes in diagnosis and patient treatment in an unselected memory clinic cohort: the ABIDE project. JAMA Neurol. https://doi.org/ 10.1001/jamaneurol.2018.1346

55. de Wilde A, Ossenkoppele R, Pelkmans W et al (2019) Assessment of the appropriate use criteria for amyloid PET in an unselected memory clinic cohort: the ABIDE project. Alzheimers Dement. https://doi.org/10.1016/j.jalz.2019.07.003

56. Zhang S, Smailagic N, Hyde C et al (2014) 11CPIB-PET for the early diagnosis of Alzheimer's disease dementia and other dementias in people with mild cognitive impairment $(\mathrm{MCl})$. Cochrane Database Syst Rev. https://doi.org/10.1002/ 14651858.CD010386.pub2

\section{H. Remschmidt, K. Becker (Hrsg.) Kinder- und Jugendpsychiatrie und Psychotherapie}

Stuttgart: Thieme (Verlag) 2020, 7, 560 S., (ISBN: 978-3-13-241122-7), 99,99 EUR

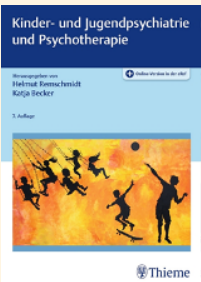

Die vorliegende 7 . Auflage des bewährten Lehrbuchs erfuhr eine vollständige Überarbeitung und Aktualisierung. In enger

Zusammenarbeit zwischen dem früheren Lehrstuhlinhaber der Marburger Klinik und seiner Nachfolgerin Frau Becker wurden alle Kapitel auf den neuesten Stand gebracht, das interdisziplinäre Autorenteam erweitert und folgende Kapitel neu aufgenommen: Schlafassoziierte Störungen, Schulabsentismus, Posttraumatische Belastungsstörungen, Suizidalität, nicht-suizidales selbstverletzendes Verhalten sowie Prävention. Das Ergebnis ist ein hoch informatives, umfassendes und dennoch übersichtliches Lehrbuch, das den aktuellen Wissensstand des Fachgebiets eindrucksvoll und überzeugend darstellt und nicht nur rasche Orientierung bietet, sondern auch vertieftes Wissen.

Zu Beginn wird dem Leser ein grundlegendes Verständnis für die psychische und körperliche Entwicklung des Kindes, relevante Entwicklungskonzepte, Informationen zu entwicklungspathologischen Belastungen und Risikofaktoren sowie Modellvorstellungen zu Ätiologie und Genese vermittelt (Teil I).

Es schließen sich umfangreiche Hinweise zur Anamnese-Erhebung, zur körperlich-neurologischen Untersuchung, zur Beurteilung des psychischen Befundes sowie zur testpsychologischen, neuropsychologischen, Familien-, apparativen und Labordiagnostik an. Eigene Beiträge setzen sich mit dem diagnostischen Prozess sowie der Klassifikation psychischer Störungen auseinander, so dass der Leser die verschiedenen Untersuchungsschritte und die damit verbundenen aufwändigen und differenzierten Interventionen sehr konkret nachvollziehen kann (Teil II).

Im Zentrum des Lehrbuches und mit dem größten Umfang (fast 300 Seiten) werden die psychischen Störungen und Erkrankun- gen differenziert und präzise dargestellt. Die einzelnen Kapitel folgen einer einheitlichen Systematik. Einleitend wird eine Definition und Klassifikation der jeweiligen Symptomatik vorgenommen, es schließen sich Angaben zur Epidemiologie, Diagnostik und Differenzialdiagnostik, zu komorbiden Störungen, zur Ätiologie und Genese sowie zur Therapie an. Zur besseren Anschaulichkeit finden sich jeweils mehrere typische Fallbeispiele und darüber hinaus Hinweise zur relevanten weiterführenden Literatur.

Ein eigener Abschnitt (Teil IV) widmet sich in acht Kapiteln der Therapie, Rehabilitation und Prävention. Neben allgemeinen Gesichtspunkten, die es in der Behandlung von Kindern und Jugendlichen zu beachten gibt, werden spezielle Aspekte wie Elterntraining, medikamentöse Behandlung, kombinierte Therapieformen, Rehabilitation und Prävention abgehandelt.

Der Abschluss des Lehrbuchs (Teil V) bietet Informationen zu den rechtlichen und institutionellen Voraussetzungen, zur gerichtlichen Kinder- und Jugendpsychiatrie, wobei neben wichtigen Hinweisen zur Rechtsstellung des Kindes und Jugendlichen spezielle Vorgehensweisen und Fragestellungen der Begutachtung aufgegriffen werden.

Es ist den Herausgebern und Autoren beeindruckend gelungen, das aktuelle Wissen über die Grundlagen, die Diagnostik und Therapie von psychischen Störungen im Kindes- und Jugendalter umfangreich und dennoch übersichtlich darzustellen. Das Buch ist deshalb Allen nachdrücklich zu empfehlen, die sich über seelische Erkrankungen, deren Ursachen und Folgen und ihre Behandlung im Kindes- und Jugendalter informieren oder ihr Wissen vertiefen wollen.

Gerd Lehmkuhl (Berlin) 
Hier steht eine Anzeige.

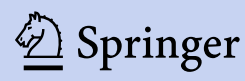

